\title{
Design and study the performance of optical nanoantenna
}

\author{
Gufran A.Hassan ${ }^{a^{*}}$ and Jawad A.Hassan ${ }^{a}$ \\ ${ }^{a}$ Department of electronic and communication engineering, University of Baghdad Institute of Laser for Postgraduate Studies, baghdad -iraq
}

\author{
Article history:

\section{Keywords:} \\ Optical \\ Nanoantenna \\ S-parameter \\ Resonance Freq
}

ART ICLE INFO

Received 22 May 2019

Received in revised form 30 June 2019

Accepted 30 June 2019

\begin{abstract}
A B S TR A C T
Recently, the light metallic interaction of nanostructures has grown to be an area of intensive care research due to advances in modern fabrication techniques. Unique effects have been observed in a nanostructure, and their applications have been found in various areas like in the manipulation of light on the nanometer scale. In this paper Nanoantenna has been introduced and invetigated by studing the effect of changing its parameters such as (shape, length, thickness, and the gap distance between two nanostructures) on the response of the nanoantenna (far field directivity, optical resonance frequency, and S-parameter). Here, a bow tie antenna has been chosen and additional parameters have been considered in the simulation, such as antenna thickness and material, and substrate material. The simulations have been generated using computer simulation technology (CST) studio. Optical antenna is performed from a pair of nanoparticles brought in close nearness, these pairs are separated by small gap to make a high electric field in its gap region. This feature can be employed for biosensing or SERS to improve the detection limit and measure the presence of single molecules.
\end{abstract}

\section{Introduction}

The field of an optical nanoantenna is a quickly developing area, optical antenna is formulated and major characteristics relevant to this structure are identified. A class of nanoantennas, metallic and dielectric are made and discussed. The study of techniques and optical phenomena in the nanometer (nm) scale are called a nano-optics [1]. Nanoantennas are used to convert electromagnetic wave radiation into electric current and vise versa, so nanoantennas are the same of antennas at radio frequencies and microwave [1]. The radio frequency (RF) antenna is a good technology able to transmit electromagnetic wave that is localized within a great sub-wavelength [2]. But in the operation of the nanoantennas AT a visible wavelength different from (RF) antennas and should be corrected the design and analysis rules to the nanoantennas [1]. So, the antennas could work in the visible of the electromagnetic spectrum when the dimension of the antenna is decreased to a nanometer range $(\mathrm{nm})$ [3], and the frequency is at (hundreds of terahertz) high frequency to operate in visible light. The earlier proposed antennas which are related to the geometric effects on the response of nanoantenna were Yu-Ming, W., Le-Wei, L., \& Bo, L. (2010, April), [4].
When changing the gap length of the bowtie nanoantenna from $10 \mathrm{~nm}$ to 40 $\mathrm{nm}$ the closer arrangement for an antenna with narrower gaps tends to exhibit higher light intensity. The intensity at resonance for nanoantenna with the separation gap of $10 \mathrm{~nm}$ is much higher than those with a gap of $40 \mathrm{~nm}$. Murad, Fadel A., Faris Mohammed Ali, and Ali Azzawi Hassan. (2016):[5] noticed that the change of the length of optical nanoantenna affects on the electrical fields in a direction of propagation waves $(x, y)$, that shows approximately linear behavior. So, the increased of the face area of the optical nanoantenna to increase the length decreasing the electric field in the gap for two electric fields in $\mathrm{Y}$ direction (Ey) and $\mathrm{X}$ direction (Ex), which led to distribute charges over a larger area, and therefore led to less concentration of charge points of convergence. There are many applications of nanoantenna such as, medical aaplications (diagnosis, drug delivery, textile engineering), chemical and environmental applications (stimulus), and electronics \& communications (semiconductor devices, optical devices).

* Corresponding author.

E-mail address: gofabdh@gmail.com (Gufran A.Hassan) 


\section{Design of The Optical Nano Antenna}

The optical nanoantenna has been designed with a thickness of $20 \mathrm{~nm}$ representing the total thickness of the constituent layers which consist of 2 layers (gold $\mathrm{Au}$ - silicon dioxide $\mathrm{SiO} 2$ ). The first proposed antenna consists of flat edge bow-tie structure and the other side is tip with a width $\mathrm{W}=(140$ ) $\mathrm{nm}$, thickness $\mathrm{T}=(20) \mathrm{nm}$, a length $\mathrm{L}=(130) \mathrm{nm}$ gap distance $\mathrm{g}=(10) \mathrm{nm}, \alpha=\left(64.94^{\circ}\right)$ and the material is (gold a lossy metal) without substrate and in the second section these nanoantenna are printed on a substrate silicon (normal) Material Set as a Default Type Normal Epsilon $=11.9, \mathrm{Mu}=1$, Electric cond $=0.00025[\mathrm{~S} / \mathrm{m}]$, Rho $=2330$ $\left[\mathrm{kg} / \mathrm{m}^{\wedge} 3\right]$, Thermal conductivity $=148[\mathrm{~W} / \mathrm{K} / \mathrm{m}]$, Thermal expansion $=5.1$ [1e-6/K], and with a width $\mathrm{w}=(200) \mathrm{nm}$, length $\mathrm{L}=(290) \mathrm{nm}$, thickens $\mathrm{T}$ $=(20) \mathrm{nm}$ as shown in Fig. 1. The substrate ahs been used with the nanoantenna because of Nano-antennas have the capability to detain light down to the nanoscale. Due to their plasmon resonance properties a huge field intensities, a number of instructions of magnitude upper than the incident one, have been achieved within these structures, allowing to study of the light-matter interaction in a nanoscale. One substantial achievement is that the optical resonances can be tuned with dielectric constant and geometrical form of the nanostructures and also with the illumination properties (polarization, wavelength) and so on. The overlap between the intense subwavelength resonant mode and diffraction limited incoming waves is in general weak, alternate solutions were recently proposed to improve light conjugation into the plasmonic nanostructures. These solutions depend on the conjunction between optical dielectric resonators of higher quality factors and nanoantenna [6]. When the current hardly flows through the material. Therefore, these materials are a higher conductivity. So, the conductivity is the power loss in the material. Lossy metal is defined as material with good conductivity when the material conductivity is more than zero [7].

$j=\sigma E$

$\mathrm{J}$ is the current density

$\mathrm{E}$ is the electric field

$\sigma$ conductivity

Electromagnetic Simulator is used to design the antenna and to do numerical analysis. The proposed antenna is capable of concentrating the electric field in the side of metal of the bow-tie by a discrete port with incident frequency $\mathrm{f}=(0-700) \mathrm{THz}$. To apply the explanation of boundary value problems in engineering (FEM) method a computational technique is used. The boundary problems are known as (a field problem), the changeable field depends on the interest it is controlled by the differential equation [8]. The boundary condition is the specific standards of the field variables on the boundary of the field [8]. So, the optical problems would be solved by Maxwell's equations [11], by using the harmonic approaches [9].

$E(t)=E_{\mathrm{o}} e^{-i w t}$

With no outer charge or current $\rho=J=0$

It can apply the equation of an electric field [9],

$\nabla \times M^{-1} \nabla \times E-\omega^{2} \varepsilon E=0$

$\nabla \cdot \varepsilon E=0$

This antenna is designed to study the electromagnetic radiation (far field directivity, S -parameter, radiation pattern ) which is analysized with a linear polarized in this simulation. When using numerical settling of Maxwell's equation in the electromagnetic simulation, a good type of mesh affects on the simulation speed and precision. So,( hexahedral) mesh grid is considered where the density of the mesh is determined by the cell size and cells per wavelength are given as 20 . The number of largest cell mesh is measured as 71.5657 and the number of cells is 21,120, Excitation of surface plasmon oscillation occurs due to the impinging of visible or infrared light on the antenna surface which creates hot spots where the field intensity become enhanced and drives the current towards the feeding point of the antenna, By using these features, the antenna can be designed for many applications.

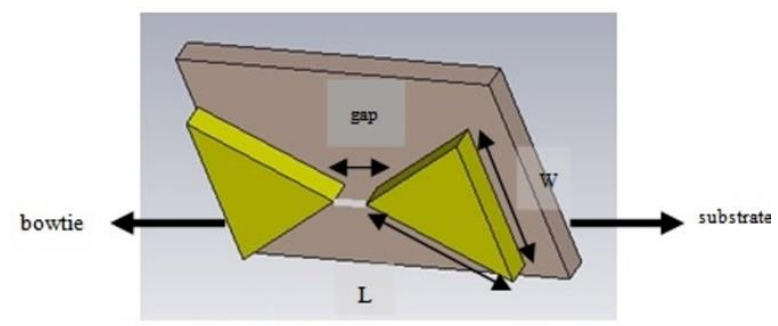

Figure (1) optical bowtie nanoantenna with a substrate

\section{Radiation pattern}

An antenna radiation pattern is defined as a mathematical function of the radiant personality of an antenna as a meaning of space coordinates are known as (radiation pattern) It is calculated on the area of the far-field and it is pictured as a function of a directional coordinate [10]. As shown in Figs. 2 and 3, the bow tie antenna has a (donut) form radiation pattern; in the donut form throw the z-axis, which would identify to the radiation straight overhead the antenna so, a very small power will be transmitted. But throw the $\mathrm{x}-\mathrm{y}$ plane the transmitted power is maximum [11].

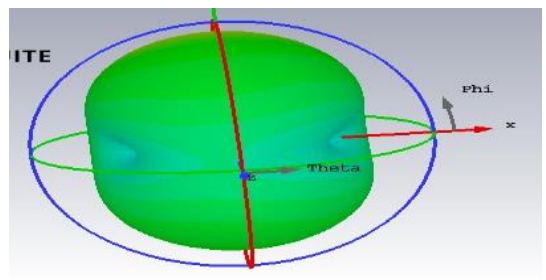

Figure 2 3-d radiation pattern for optical bowtie nanoantenna with a length $L=(130) \mathrm{nm}$, width $W=(140) \mathrm{nm}, \alpha=\left(64.94^{\circ}\right)$, thickness $\mathbf{T}=(20) \mathrm{nm}$, gap distance $\mathrm{g}=(\mathbf{1 0}) \mathrm{nm}$, material is (gold a lossy metal) this nanoparticles are with a silicon (normal) substrate with $\mathrm{w}=(200)$ $\mathrm{nm}, \mathrm{L}=(290) \mathrm{nm}, \mathrm{T}=(20) \mathrm{nm}$, frequency $=(650) \mathrm{THz}$

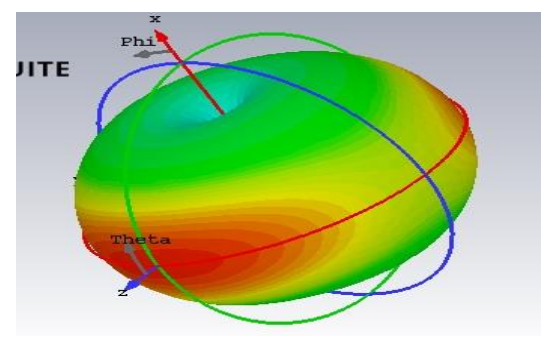

Figure 3 3-d radiation pattern for optical bowtie nanoantenna with a length $L=(130) \mathrm{nm}$, width $W=(140) \mathrm{nm}, \alpha=\left(64.94^{\circ}\right)$, thickness $\mathrm{T}=(20) \mathrm{nm}$, gap distance $\mathrm{g}=(\mathbf{1 0}) \mathrm{nm}$, the material is (gold a lossy metal) without substrate frequency $=(650) \mathrm{THz}$ 
The far-field directivity of this bowtie nanoantenna was calculated as shown in the Figs. 4 and 5. Farfield Directivity Abs (Phi=90)

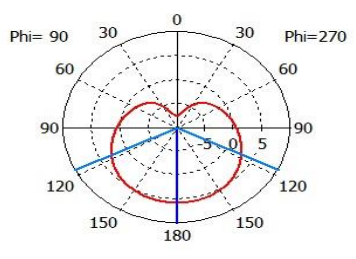

Theta / Degree vs. dBi

Figure 4 Far-field directivity for optical bow tie nanoantenna with a length $L=(130) \mathrm{nm}$, width $\mathrm{W}=(140) \mathrm{nm}, \alpha=\left(64.94^{\circ}\right)$, thickness $\mathrm{T}=(20)$ $\mathrm{nm}$, gap distance $\mathrm{g}=(\mathbf{1 0}) \mathrm{nm}$, material is (gold a lossy metal) this nanoparticls are with a silicon (normal) substrate with $\mathrm{w}=(\mathbf{2 0 0}) \mathrm{nm}$, $\mathrm{L}=(\mathbf{2 9 0}) \mathrm{nm}, \mathrm{T}=(\mathbf{2 0}) \mathrm{nm}$

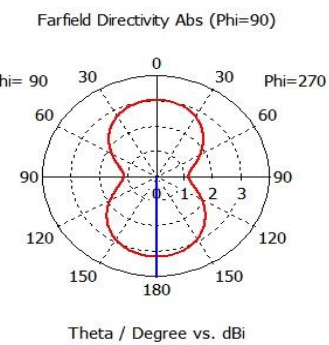

Figure 5 Far-field directivity for optical bowtie nanoantenna with a length $\mathrm{L}=(130) \mathrm{nm}$, width $\mathrm{W}=(140) \mathrm{nm}, \alpha=\left(64.94^{\circ}\right)$, thickness $\mathrm{T}=$ (20) $\mathrm{nm}$, gap distance $\mathrm{g}=(\mathbf{1 0}) \mathrm{nm}$, the material is (gold a lossy metal) these nanoparticles are without substrate At a Frequency $=650 \mathrm{Thz}$

The red curve stands for E $\theta$ pattern, the blue lines are marked for a half power bandwidth the green curve is the magnitude of the back loop the Main lobe magnitude=5.72 dB, Main lobe direction=180 deg, and The angular width (3) $\mathrm{DB}=126.8 \mathrm{deg}$. At Frequency $=650 \mathrm{THz}$ for bow tie without substrate. But for bow tie with a substrate, it was the Main lobe magnitude $=3.19 \mathrm{~dB}$ and the Main lobe direction $=180 \mathrm{deg}$.

Sometimes the antennas have a polar of (omnidirectional) which founds a radiation pattern is (isotropic) in a single plane. So, a bow tie antenna has omnidirectional pattern [11]. It shows a half power bandwidth obtained at a frequency (650 THZ) by simulation of the optical bowtie nanoantenna with and without substrate, the radiation pattern was calculated as shown in Figs. 4 and 5.

\section{Thickness effect}

In this section, the effects of the thickness on the response of nanoantenna were Studying. By simulating of optical bowtie nanoantenna with the same parameter above This can be shown in Fig. (6)(a,b,c,d,e) when change the thickness of bowtie nanoantenna as $\mathrm{T}=(50,40,30,20,10$, $)$ nm respectively, with fixed the other parameter of bowtie nanoantenna length $\mathrm{L}=(130) \mathrm{nm}$, width $\mathrm{W}=(140) \mathrm{nm}, \alpha=\left(64.94^{\circ}\right)$, gap distance $\mathrm{g}=(10) \mathrm{nm}$, material is (gold a lossy metal) without a substrate and frequency at (650)THz.

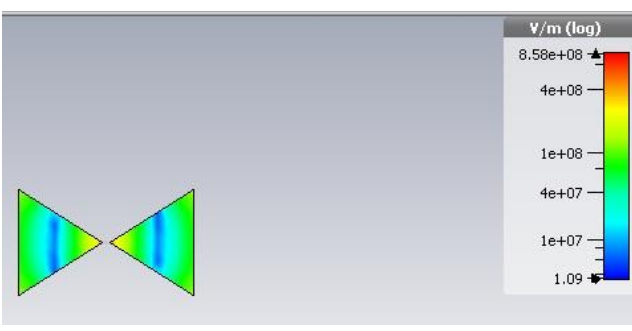

(a) $\mathrm{T}=(\mathbf{5 0}) \mathbf{n m}$

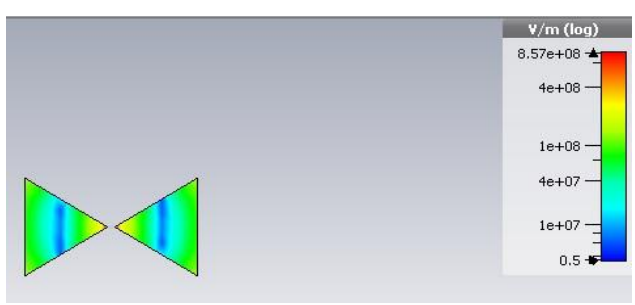

(b) $\mathrm{T}=(\mathbf{4 0}) \mathrm{nm}$

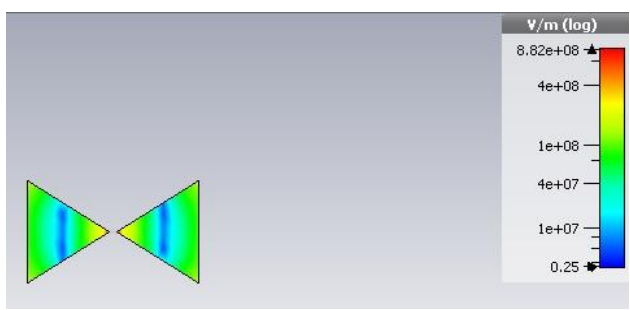

(c) $\mathbf{T}=(30) \mathrm{nm}$

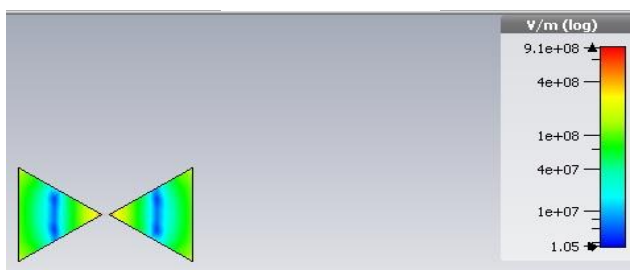

(e) $\mathbf{T}=(20) \mathbf{n m}$

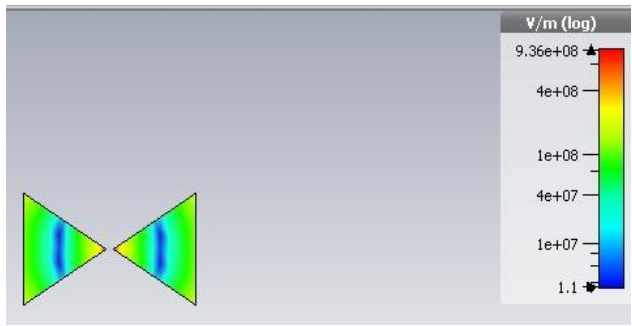

Figure (6) (d) $\mathbf{T}=(10) \mathbf{n m}$

From the results, we notice that when changed the thickness of nanoantenna the Field enhancement at the tip of the nanoantenna increase when the thickness decreases [12].

and the increasing of electric field enhancement when decreasing the thickness because of the intense coupling near -the field in thickness and this is driving to a great partial Plasmon shift [12]. As shown in Fig. (6) (a, $\mathbf{b}, \mathbf{c}, \mathbf{d}, \mathbf{e})$ the directivity increase when decreases the thickness of bow tie nanoantenna. When the thickness (50)nm the directivity is $(8.58 \mathrm{e}+0.8) \mathrm{v} / \mathrm{m}$ and when the thickness $(10) \mathrm{nm}$ the directivity is $(9.36 \mathrm{e}+0.8) \mathrm{v} / \mathrm{m}$ 


\section{Material effect}

In this section, the effect of choosing the material of nanoantenna was studying the first way was studying the effect of the coefficient of the materials used. The second way was studying the capability of the material to simulate a current as shown in Fig 7.(a-d). By dint of the radiant flux incident on the nanoantenna [13]. It was studying the different material used in the design of bowtie nanoantenna with a fixed of other parameters (length, gap distance, thickness). the result was as shown in Table 1.

Table 1 result of optical bowtie nanoantenna with a different material

\begin{tabular}{llll}
\hline Material & $\begin{array}{l}\text { S-parameter } \\
\text { (dB) }\end{array}$ & $\begin{array}{l}\text { Discrete port } \\
\text { current (dB) }\end{array}$ & $\begin{array}{l}\text { Resonance } \\
\text { frequency (THZ) }\end{array}$ \\
\hline Gold & -38.6 & -17.7 & 367 \\
Nickel & -2.68 & -28 & 145.4 \\
Silver & -31.3 & -16.8 & 369.4 \\
Aluminum & -37.9 & -17 & 367 \\
\hline
\end{tabular}

Table 1. shows the resonance frequency, S- parameter, and current response for different material are used in a simulation design of bowtie nanoantenna with width $\mathrm{W}=(140) \mathrm{nm}$, a length $\mathrm{L}=(130) \mathrm{nm}, \alpha=\left(64.94^{\circ}\right)$ ,thickness $\mathrm{T}=(20) \mathrm{nm}$, gap distance $\mathrm{g}=(10) \mathrm{nm}$, the material is (gold ,nickel,silver,and aluminum) without a substrate the results were changed depending on the optical properties of the materials used. So, it is very important to choose the properly material in the design to getting good efficiency of bowtie nanoantenna [13].

\section{Gap effect}

Antenna theory provides the improvement of the electric field (E-field) in close proximity to a sharp end metal spot. So, antennas have been making a resonant in (IR) and microwave region when it is designed as a nanoscale metallic with a sharpened will progress locally enhanced (E-field)[14]. So, when fabricated two a nanoscale triangles tip to tip metallic with a small distance gap between them this is a bowtie nanoantenna which gives a high electric field intensity in the air of the gab $[14,15]$. Because of a localized surface Plasmon excitation [15] So, by varying the gap distance between the two nanostructures we can be controlling the enhancement of electric field around the gap, and the resonance frequency, s-parameter, and wavelength. This can be shown in fig 8 by simulation of a bow tie nanoantenna with a length $\mathrm{L}=(130) \mathrm{nm}$, width $\mathrm{W}=(140) \mathrm{nm}, \alpha=\left(64.94^{\circ}\right)$, thickness $\mathrm{T}=(20) \mathrm{nm}$, gap distance changed as $\mathrm{g}=(30,25,20,15,10,5)$ material is (gold a lossy metal) this nanoparticles are printed on a silicon (normal) substrate with $\mathrm{w}=(200) \mathrm{nm}, \mathrm{L}=(290) \mathrm{nm}, \mathrm{T}=(20) \mathrm{nm}$, This result shows a correlation between the gap and resonance frequency when the light is parallel polarized to the axis along the pairs that combine to the nanoparticle center [14]. As shown in table (2), at a shorter gap the resonance frequency is increasing and the spectra sharply a blue-shift. But, at the long gap the resonance frequency is decreasing and the spectra sharply to a redshift And this distance gap effect too on s-parameter (reflection coefficient). As shown in Fig 8.

Table (2) result of optical bowtie nanoantenna with a substrate and with a different distance gap

\begin{tabular}{llll}
\hline $\begin{array}{l}\text { Gap } \\
(\mathbf{n m})\end{array}$ & $\begin{array}{l}\text { Resonance } \\
\text { frequency(THZ) }\end{array}$ & $\begin{array}{l}\text { S-parameter } \\
(\mathbf{d B})\end{array}$ & $\begin{array}{l}\text { Wavelength } \\
(\mathbf{n m})\end{array}$ \\
\hline 30 & 310.23 & -16.9 & 966.4 \\
25 & 320.45 & -17.8 & 935.54 \\
20 & 330.68 & -19.11 & 906.59 \\
15 & 343.18 & -20.97 & 873.57 \\
10 & 356.82 & -24.1 & 840 \\
5 & 377.3 & -33 & 795 \\
\hline
\end{tabular}

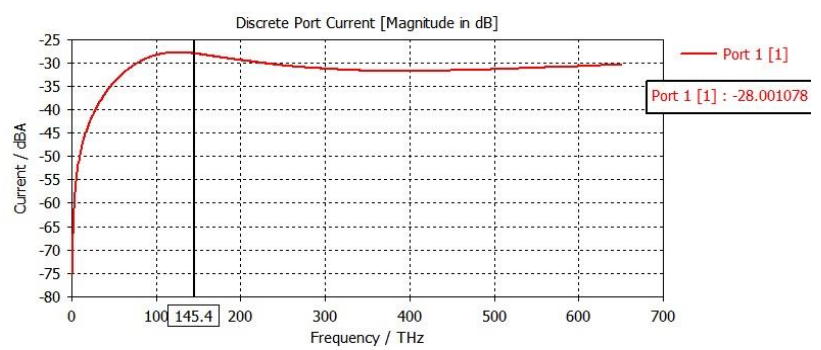

(a) For aluminum

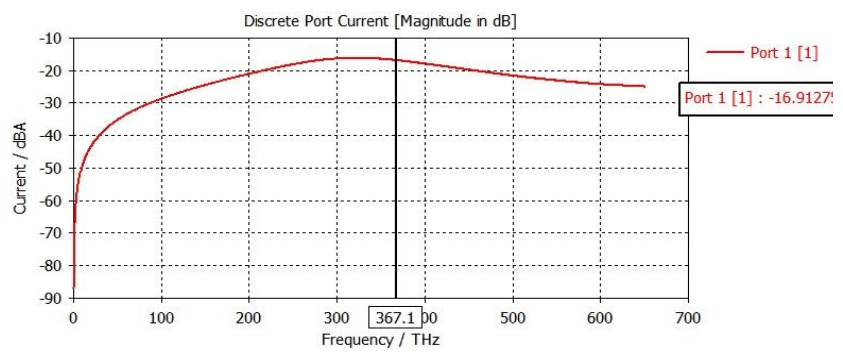

(b) For silver

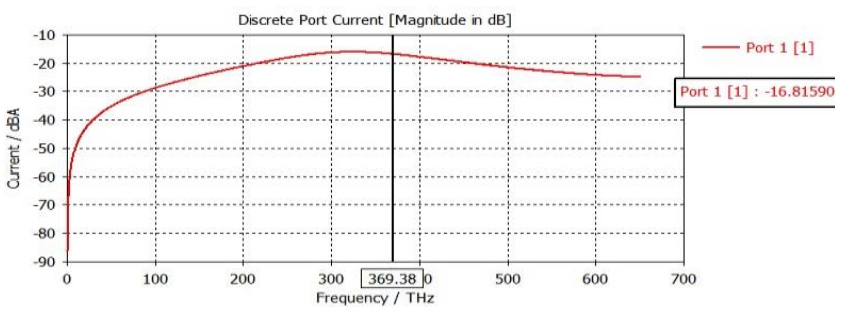

(c) For Nickel

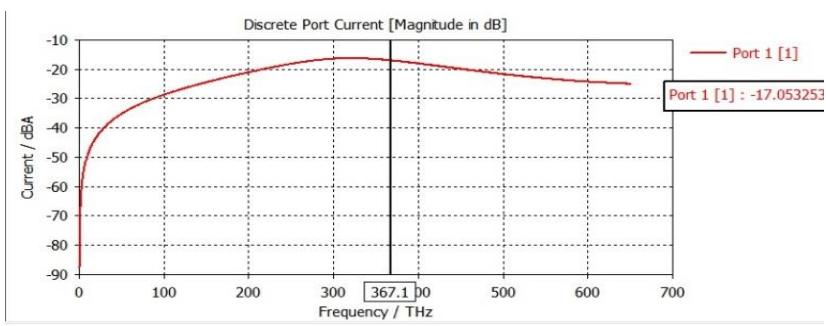

(d) For Nickel

Figure 7 Shows the current (db) vs. Frequency (THz) for different materials.

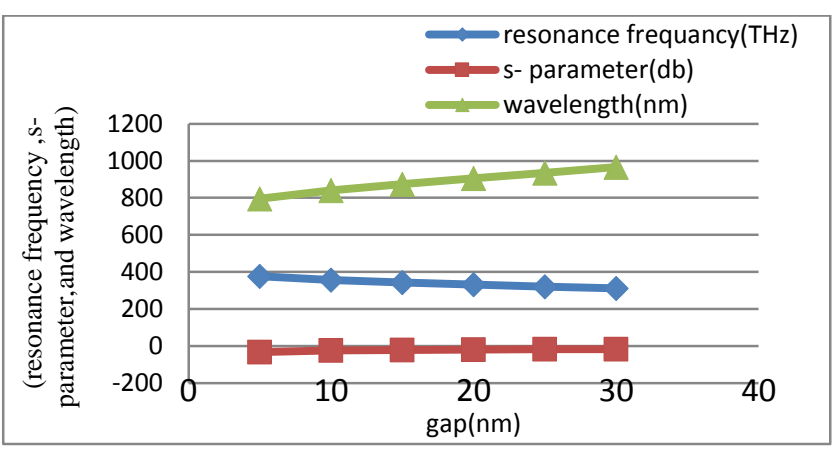

Figure 8 effects of change the gap of the nanoantenna with substrate on the ( resonance frequency, s-parameter,and wavelength). 
These steps of simulated were repeated with the same optical bowtie nanoantenna without substrate surely it is the same effect of bowtie nanoantenna with a substrate we have, but the results of bowtie nanoantenna without substrate are the best for the resonance frequency and optical wavelength. As shown in Fig. 9. and the result in a Table 3.

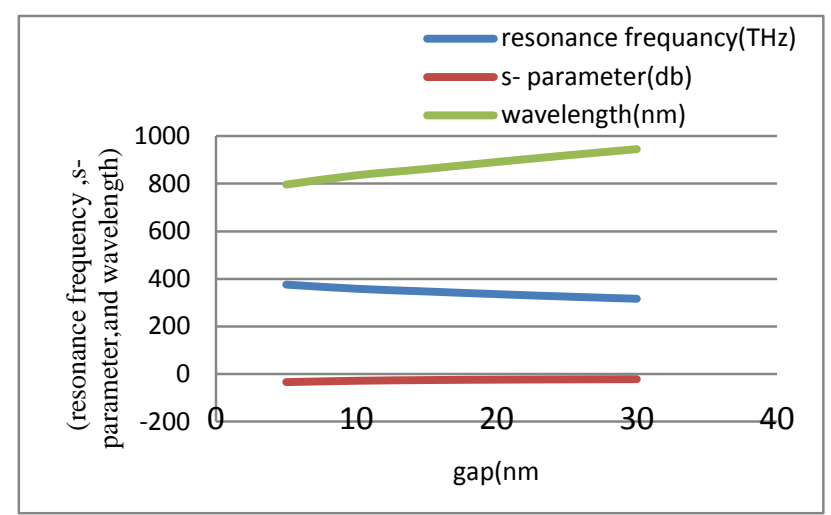

Figure 9 effects of change the gap of the nanoantenna without substrate on the( resonance frequency,s-parameter,and wavelength)

Table (3) result of optical bowtie nanoantenna without a substrate and with a different distance gap

\begin{tabular}{llll}
\hline $\begin{array}{l}\text { Gap } \\
\text { (nm) }\end{array}$ & $\begin{array}{l}\text { Resonance } \\
\text { frequency(THZ) }\end{array}$ & $\begin{array}{l}\text { S-parameter } \\
\text { (dB) }\end{array}$ & $\begin{array}{l}\text { Wavelength } \\
\text { (nm) }\end{array}$ \\
\hline 30 & 317 & -22.5 & 945.57 \\
25 & 326.14 & -23.3 & 919.21 \\
20 & 336.36 & -24.4 & 891.28 \\
15 & 347.7 & -26.18 & 862.2 \\
10 & 359.1 & -29 & 835.08 \\
5 & 376.6 & -34.7 & 796.05 \\
\hline
\end{tabular}

\section{S-parameter S11}

S-parameter is representing the amount of the power that reflected from the antenna It is could write as a gamma $(\Gamma)$ called return loss, [11]. When $\mathrm{S}$-parameter $\left(\mathrm{S}_{11}\right)$ is $=-10 \mathrm{~dB}$ this is mean if $3 \mathrm{~dB}$ of power is reached to the antenna then $-7 \mathrm{db}$ will be reflected and The rest of the power was accepted to the antenna, this accepted power is each emit or absorb as a wounded within the antenna. If $\left(\mathrm{S}_{11}\right)$ is $0 \mathrm{db}$ this meaning no power is radiated all power will be reflected from the antenna.

Fig. 10. (a-f) shows the results of S-parameter of optical bowtie nanoantenna which show in Fig. 1. with a substrate when changing the distance gap $\mathrm{g}=(30,25,20,15,10,5) \mathrm{nm}$ respectively.

Fig. 11. shows the results of s-parameter of optical bowtie nanoantenna which show in Fig. 1. without a substrate) when changing the distance gap $\mathrm{g}=(30,25,20,15,10,5) \mathrm{nm}$ respectively, from Figs. 10. and 11. we notice that s-parameter is a function of the gap, decreasing gap leads to increasing the reflection coefficient of the nanoantenna.

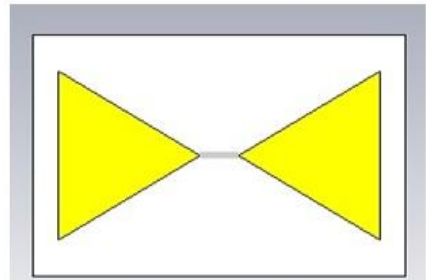

(a) $\mathrm{g}=(\mathbf{3 0}) \mathrm{nm}$

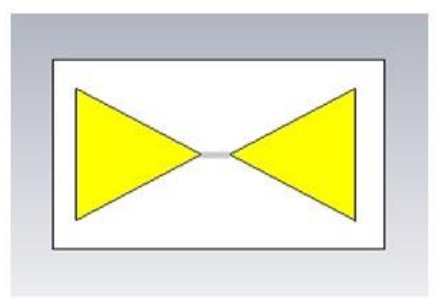

(b) $\mathbf{g}=(\mathbf{2 5}) \mathrm{nm}$

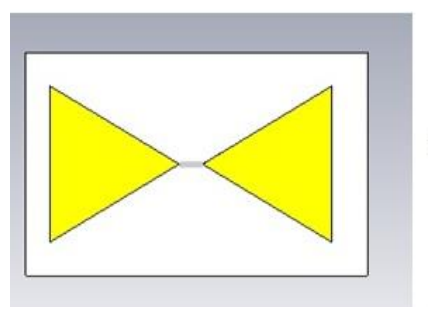

(c) $\mathbf{g}=(\mathbf{2 0}) \mathrm{nm}$

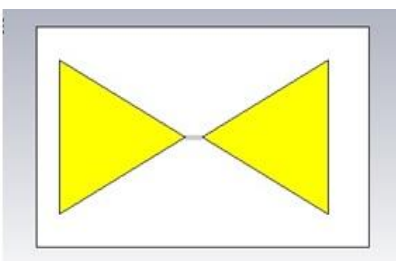

(d) $\quad \sigma=(15) \mathrm{nm}$

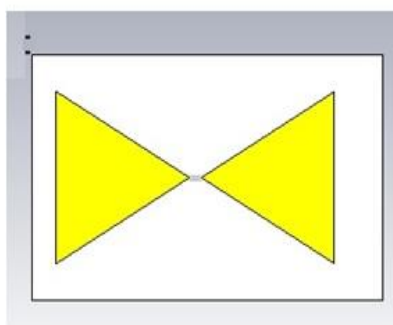

(e) $\mathrm{g}=(\mathbf{1 0}) \mathrm{nm}$

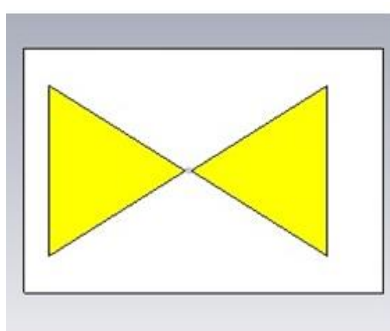

(f) $\mathbf{g}=(\mathbf{5}) \mathbf{n m}$

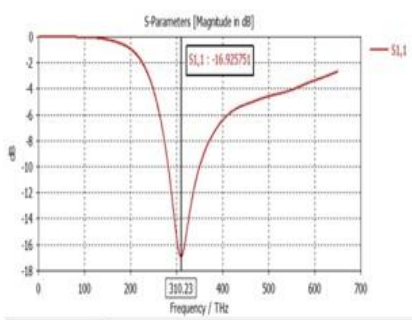

s-parameter for $\mathrm{g}=(\mathbf{3 0}) \mathrm{nm}$

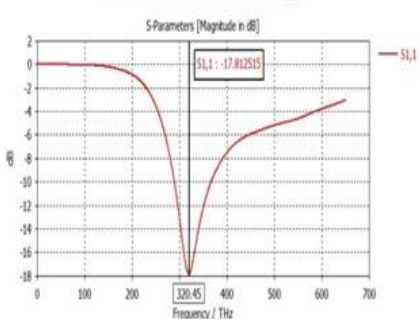

-parameter for $\mathbf{g}=(\mathbf{2 5}) \mathrm{nm}$

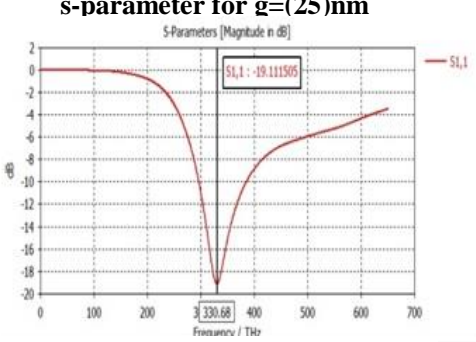

s-parameter for $\mathrm{g}=(\mathbf{2 0}) \mathrm{nm}$
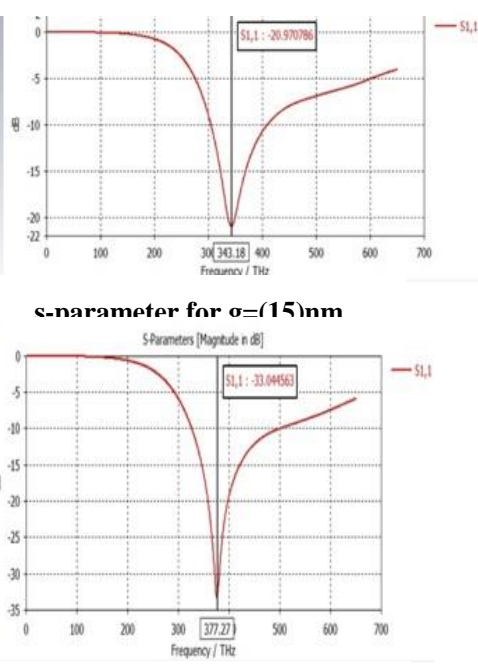

s-parameter for $\mathrm{g}=(\mathbf{1 0}) \mathrm{nm}$

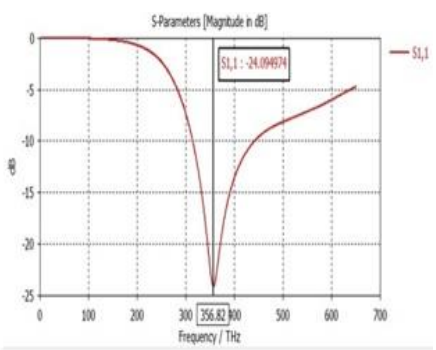

s-parameter for $\mathrm{g}=(\mathbf{5}) \mathrm{nm}$
Figure 10 the results of S-parameter of optical bowtie nanoantenna 

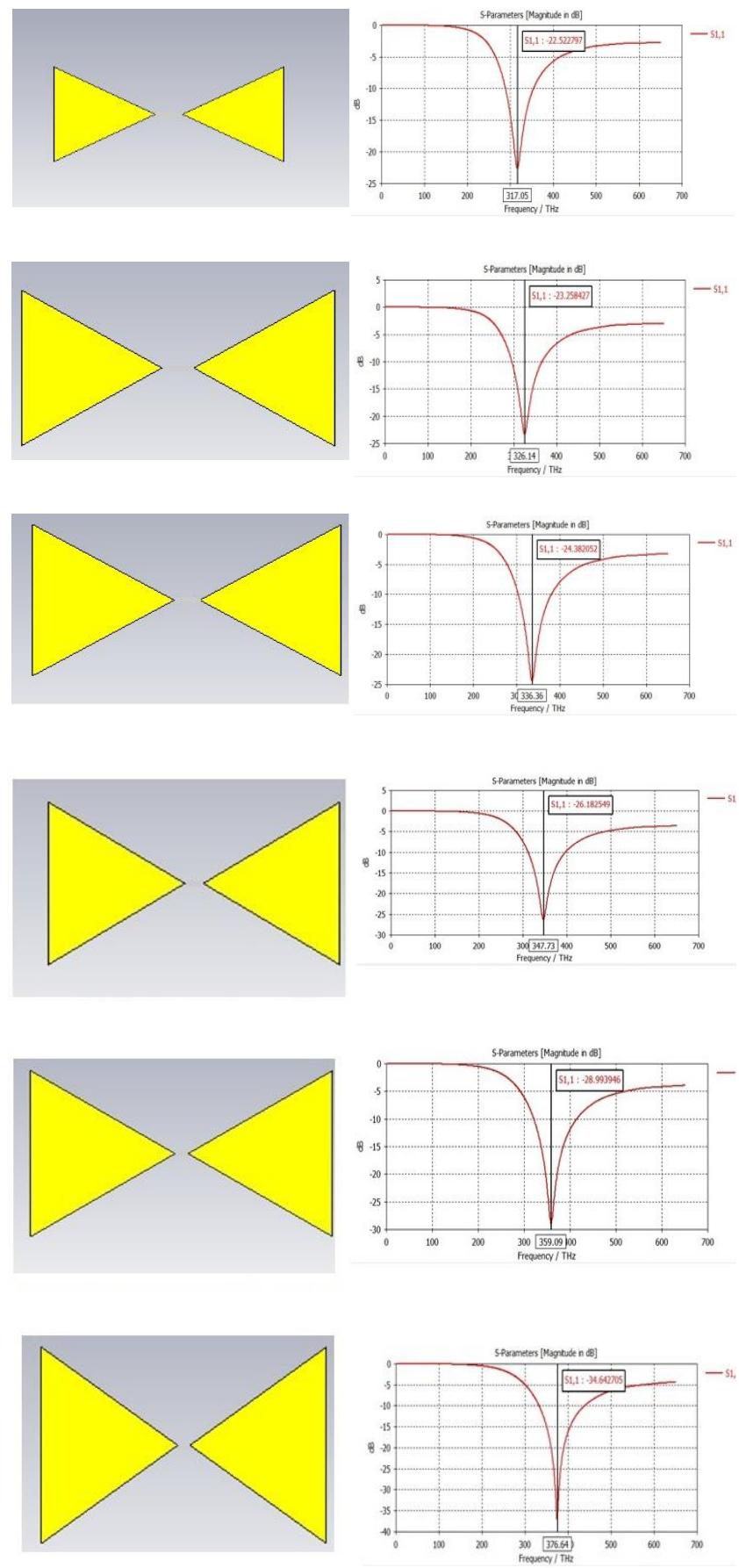

\section{Conclusion}

1- Optical antennas are the general tool for the handling of light on the scale of a nanometre and they are also able to control a farfield area. Present optical antenna research is being aggravated in special by expansion in nanofabrication technology and RF antenna analogies.

2- Change in the parameter of optical nanoantenna, such as gap distance, effects on the resonance frequency and the Sparameter.
3- Decreasing the distance gap between two nanostructures of a bowtie antenna leads to increase the resonance frequency and Sparameter and a highly shift in the wavelength.

4- Increasing the distance gap between two nanostructure of a bowtie antenna leads to decreasing the resonance frequency and S-parameter and a high redshift wavelength.

5- When decreasing the thickness of the bowtie antenna this leads to increase enhancement of the electric field and increasing the directivity in the tip of the antenna.

6- when changing the material of bowtie antenna the results are effected because of the change of the material optical properties effects on the resonance frequency with cause a shift and change the induced current.

So by controlling the parameter of a bowtie nanoantenna, we can have the best result as we need, this bow tie nanoantenna can be the best to use in the optical application if used by incident electric field frequency at $(0-$ $700 \mathrm{THz})$ the wavelength is $(600-900) \mathrm{nm}$, so, study the effect of all these parameters of nanoantenna on the response of the antenna together can help the research to choose the design which needs for any application with a special parameter and spatial resonance frequency or reflection coefficient.

\section{REFERENCES}

[1] Novotny, Lukas, and Bert Hecht. Principles of nano-optics. Cambridge university press, 2012.

[2] Alu, Andrea, and Nader Engheta. "Theory, modeling and features of optical nanoantennas." IEEE Transactions on Antennas and Propagation 61.4 (2013): 1508-1517

[3] Scholder, Olivier. Fabrication, simulation and characterization of tunable plasmonic nano antennas. Diss. ETH Zurich, 2014.

[4] Yu-Ming, W., Le-Wei, L., \& Bo, L. (2010, April). Geometric effects in designing bow-tie nanoantenna for optical resonance investigation. In 2010 Asia-Pacific International Symposium on Electromagnetic Compatibility (pp. 1108-1111). IEEE.

[5] Murad, Fadel A., Faris Mohammed Ali, and Ali Azzawi Hassan. "Study the Effect of Change the Antenna Length on the Performance of Optical Nano-Antenna Using Plasmonic Surfaces." journal of kerbala university 14.2 (2016): 124-139.

[6] El Eter, Ali, et al. "Huge light-enhancement by coupling a bowtie nano-antenna's plasmonic resonance to a photonic crystal mode." Optics express 22.12 (2014): 14464-14472.

[7] Blythe, A. R. "Electrical resistivity measurements of polymer materials." Polymer Testing 4.2-4 (1984): 195-209.

[8] Grandin, Hartley. Fundamentals of the finite element method. New York: Macmillan, 1986.

[9] Manley, Phillip, et al. "Design principles for plasmonic nanoparticle devices." Progress in Nonlinear Nano-optics. Springer, Cham, 2015. 223-247.

[10] Balanis, Constantine A. Antenna theory: analysis and design. John wiley \& sons, 2016.

[11] Choma, John. "Scattering parameters: Concept, theory, and applications." Univ. Southern California, Los Angeles, CA, USA, Tech. Rep. EE 541.2 (2006).

[12] Yang, Jie, et al. "Optimizing the bowtie nano-antenna for enhanced Purcell factor and electric field." Progress In Electromagnetics Research 44 (2014): 93-99.

[13] Mora-Ventura, Brhayllan, et al. "Responsivity and resonant properties of dipole, bowtie, and spiral Seebeck nanoantennas." Journal of Photonics for Energy 6.2 (2016): 024501.

[14] Fromm, David P., et al. "Gap-dependent optical coupling of single "bowtie" nanoantennas resonant in the visible." Nano letters 4.5 (2004): 957-961.

[15] Khaleque, Abdul, et al. "Integration of bow-tie plasmonic nano-antennas on tapered fibers." Optics express 25.8 (2017): 8986-8996. 